\title{
Levitation-Free Vibrated Droplets: Resonant Oscillations of Liquid Marbles
}

\author{
G. McHale, ${ }^{*}{ }^{\dagger}$ S. J. Elliott, ${ }^{\dagger}$ M. I. Newton, ${ }^{\dagger}$ D. L. Herbertson, ${ }^{\dagger}$ and K. Esmer ${ }^{\ddagger}$ \\ School of Science and Technology, Nottingham Trent University, Clifton Lane, Nottingham NG11 8NS, \\ United Kingdom, and Department of Physics, Kocaeli University, 41300 Kocaeli, Turkey
}

Received September 14, 2008. Revised Manuscript Received October 18, 2008

\begin{abstract}
A spherical conducting droplet in an alternating electric field is known to undergo shape oscillations. When the droplet is supported by a substrate, the shape is no longer a complete sphere, but shape resonances are still observed. To obtain a completely spherical droplet, some kind of levitation is needed, unless the droplet is in microgravity, and this has previously been provided by gas films or magnetic or other external forces. In this work, we report observations of shape oscillations of a hydrophobic-powder-coated droplet of water. A droplet of water rolled on a hydrophobic powder self-coats such that the water becomes encapsulated as a liquid marble. When the powder is a spherical hydrophobic grain with a contact angle greater than $90^{\circ}$, it adheres to the solid-water interface with more than half of its diameter projecting from the liquid, thus ensuring the encapsulated water does not come into contact with any substrate. These liquid marbles are highly mobile and can be regarded as completely nonwetting droplets possessing contact angles of $180^{\circ}$. In this work, we show that they also provide a new mechanism equivalent to levitating droplets and provide droplets with small contact areas and completely mobile contact lines for studies of shape oscillations. Liquid marbles were created using hydrophobic lycopodium and droplets of water containing potassium chloride and were excited into motion using an electrowetting-on-dielectric configuration with applied frequency swept from 1 to $250 \mathrm{~Hz}$. Both an up-and-down motion and an oscillation involving multiple nodes were observed and recorded using a high-speed camera. The resonant oscillation modes of small liquid marbles were fitted to the theory for vibrations of a free spherical volume of fluid. This work demonstrates the principle that oscillation modes of completely nonwetting droplets can be studied using a simple powder coating approach without the need for an active mechanism for levitation.
\end{abstract}

\section{Introduction}

In the absence of gravity a droplet of liquid adopts a completely spherical shape. The angular frequencies of free vibration of such an isolated droplet in a vacuum for small amplitudes is described by

$$
f_{n}^{2}=\frac{n(n-1)(n+2) \gamma_{\mathrm{LV}}}{4 \rho \pi^{2} R^{3}}
$$

where $R$ is the spherical radius, $\rho$ is the density, $\gamma_{\mathrm{LV}}$ is the surface tension, and $n$ is the mode number representing the number of nodes in the oscillation. ${ }^{1}$ To obtain a completely spherical droplet normally requires either a zero gravity or microgravity situation ${ }^{2}$ or some method of droplet levitation (magnetic, gas film, etc.). ${ }^{3,4}$ When gravity is present and a droplet is supported by a substrate, the analysis is more involved, but analytical expressions for the axisymmetric vibration of a spherical droplet in partial contact with a spherical bowl and with a fixed contact line have been derived and studied. ${ }^{5,6}$ One difference in the vibrations of such a constrained droplet compared to a free droplet is that a lowfrequency $(n=1)$ mode tending to a zero-frequency rigid body

* To whom correspondence should be addressed. E-mail: glen.mchale@ ntu.ac.uk. Phone: +44 (0)115 8483383.

${ }^{\dagger}$ Nottingham Trent University.

* Kocaeli University.

(1) Lamb, H. Hydrodynamics; Cambridge University Press: Cambridge, U.K., 1932 .

(2) Wang, T. G.; Anilkumar, A. V.; Lee, C. P. J. Fluid Mech. 1996, 308, 1-14.

(3) Perez, M.; Salvo, L.; Suéry, M.; Bréchet, Y.; Papoular, M. Phys. Rev. E 2000, 61, 2669-2675.

(4) Beaugnon, E.; Fabregue, D.; Billy, D.; Napp, J.; Tournier, R. Physica B 2001, 294-295, 715-720.

(5) Strani, M.; Sabetta, F. J. Fluid Mech. 1984, 141, 233-247.

(6) Smithwick, R. W.; Boulet, J. A. M. J. Colloid Interface Sci. 1989, 130, 588-596. displacement is predicted as the amplitude of the supported portion of the droplet reduces to zero. Creating a droplet with a small contact area and low to vanishing contact angle hysteresis is difficult on a smooth substrate even when the substrate is hydrophobic since the largest contact angles for water in air tend to be in the range $115-120^{\circ}$.

Creating shape oscillations in liquid droplets is a matter of interest for a number of reasons. At the level of liquid characterization, oscillating droplet techniques are used to measure surface tension and viscosity of liquids ${ }^{7}$ and have been proposed for measurement of contact angles. ${ }^{8}$ At the technological level, oscillations can be used to create internal mixing within sessile droplets, ${ }^{9,10}$ and indeed, surface wave induced oscillations have been deployed effectively to improve fluorescence in DNA microarrays. ${ }^{11}$ In droplet microfluidics, electric field driven oscillations have been shown to be controllable and able to create self-propelling droplets. ${ }^{12}$ It is conceivable that the combination of either substrate heterogeneity or contact angle hysteresis when coupled to droplet oscillations, however induced, could provide new methods to actuate droplet motion. In the case of droplet actuation, reducing the contact area is likely to reduce the driving force needed to initiate and sustain motion, so understanding oscillations of sessile droplets possessing small contact areas, as observed with superhydrophobic surfaces, is important.

(7) Egry, I.; Giffard, H.; Schneider, S. Meas. Sci. Technol. 2005, 16, 426-431. (8) Yamakita, S.; Matsui, Y.; Shiokawa, S. Jpn. J. Appl. Phys. 1999, 38, 31273130 .

(9) Miraghaie, R.; Sterling, J. D.; Nadim, A. NSTI-Nanotech 2006, 2, 610613.

(10) Mugele, F.; Baret, J. C.; Steinhauser, D. Appl. Phys. Lett. 2006, 88, 204106.

(11) Wixforth, A.; Strobl, C.; Gauer, C.; Toegl, A.; Guttenberg, Z. v. Anal. Bioanal. Chem. 2004, 379, 982-991.

(12) Gunji, M.; Washizu, M. J. Phys. D 2005, 38, 2417-2423. 

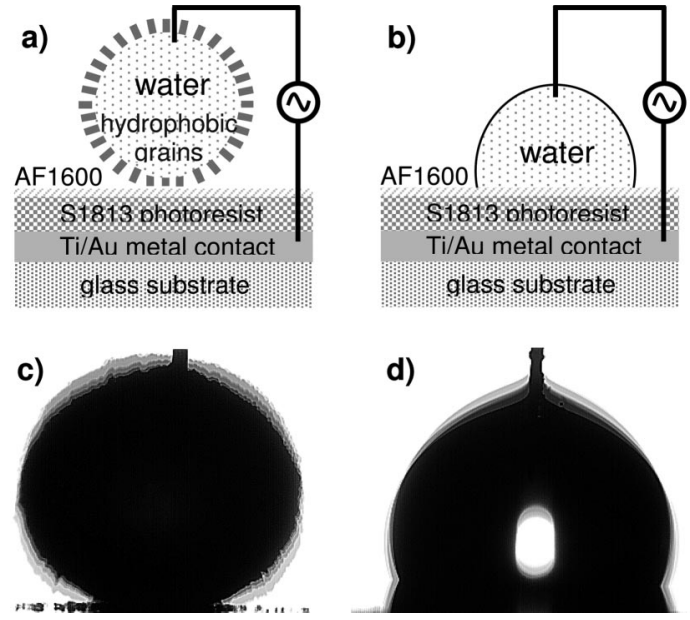

Figure 1. Configurations for inducing shape oscillations using (a) a liquid marble and (b) a hydrophobic planar surface. Associated resonances of (c) a $5 \mu \mathrm{L}$ volume liquid marble and (d) a similar volume sessile droplet on the hydrophobic flat surface. In each case, two nodes are apparent on the profile above the substrate.

In this work we consider a new approach to experimentally investigating the oscillations of droplets by using a (hydrophobic) planar surface with liquid marbles. ${ }^{13,14}$ These marbles are completely nonwetting, possess small contact areas with the substrate, and are highly mobile with zero contact angle hysteresis. In this paper, our main focus is on excitation of liquid marble shape oscillations using an ac-driven electrowetting-on-dielectric type configuration, ${ }^{15-17}$ but experiments with sessile droplets on the same substrates are considered for comparison. Image processing techniques are used to identify the resonant frequencies and show that the resonant modes of liquid marbles correspond to vibrations of substrate-supported droplets with mobile contact lines and are well-described by the theory for free spherical droplets.

\section{Experimental Method}

Liquid marbles were created by depositing droplets of $0.01 \mathrm{M}$ $\mathrm{KCl}$ in deionized water of volumes ranging from 5 to $275 \mu \mathrm{L}$ onto hydrophobized grains of lycopodium powder of size $17 \pm 3 \mu \mathrm{m}$ and "roll-coating" them as previously reported. ${ }^{17,18}$ Hydrophobization of lycopodium was achieved by immersion in cyclohexane with added hexamethyldisilizane for $48 \mathrm{~h}$ and then drying in an oven. For the oscillation experiments the marbles were placed onto a glass substrate that contained a sputter-coated Ti/Au electrode with a spincoated polymer overlayer of Shipley S1813 photoresist (thickness $2.5 \mu \mathrm{m}$ and baked at $100{ }^{\circ} \mathrm{C}$ for $60 \mathrm{~min}$ ) and capped by a $1.3 \mu \mathrm{m}$ hydrophobic layer of amorphous Teflon AF1600 (DuPont Polymers). A hydrophobized contact wire of approximate diameter $0.1 \mathrm{~mm}$ was placed into the apex of the marble and an ac voltage applied by feeding the output of an Agilent 33220A waveform generator through a Trek PZD700 amplifier (Figure 1a); Oh et al. have recently reported a study of sessile droplet oscillations using a similar configuration of a Teflon AF1600 substrate and ac electrowetting-on-dielectric excitation. ${ }^{19}$ In our case, the typical applied voltage was $200 \mathrm{~V}$ peak-to-peak with a frequency sweep from 1 to $250 \mathrm{~Hz}$ in steps of $5 \mathrm{~Hz} \mathrm{~s}^{-1}$. To more accurately identify the fundamental frequencies

(13) Mahadevan, L.; Pomeau, Y. Phys. Fluids 1999, 11, 2449-2453.

(14) Aussillous, P.; Quéré, D. Nature (London) 2001, 411, 924-927.

(15) Berge, B. C. R. Acad. Sci., Ser. II 1993, 317, 157-163.

(16) Mugelé, F.; Baret, J. C. J. Phys.: Condens. Matter 2005, 17, R705-R774.

(17) Newton, M. I.; Herbertson, D. L.; Elliott, S. J.; Shirtcliffe, N. J.; McHale, G. J. Phys. D 2007, 40, 20-24.

(18) McHale, G.; Herbertson, D. L.; Elliott, S. J.; Shirtcliffe, N. J.; Newton, M. I. Langmuir 2007, 23, 918-924.

(19) Oh, J. M.; Ko, S. H.; Kang, K. H. Langmuir 2008, 24, 8379-8386.

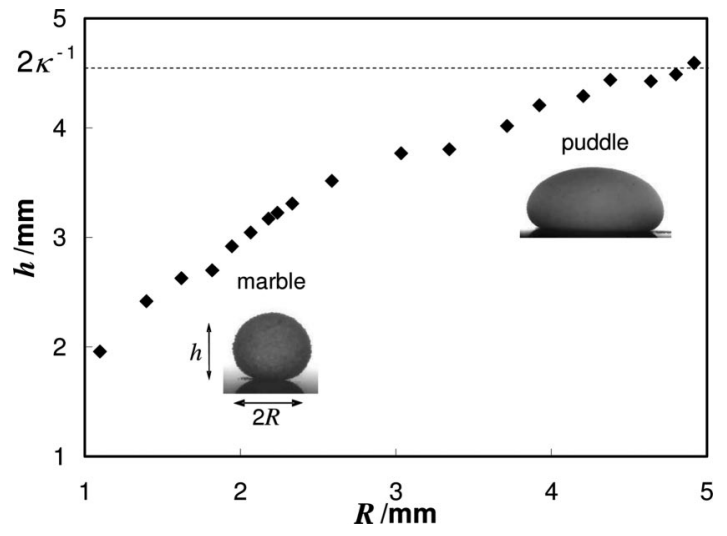

Figure 2. Height as a function of the planar radius for freshly created marbles showing the transition from marble to puddle with increasing volume. The limiting value of the puddle height gives twice the capillary length, $\kappa^{-1}$. Inset are images taken for a marble of radius $1.1 \mathrm{~mm}$ (volume $5 \mu \mathrm{L}$ ) and a puddle of radius $4.8 \mathrm{~mm}$ (volume $250 \mu \mathrm{L}$ ).

for the full marble volume range, frequency sweeps across a narrower band of width $10 \mathrm{~Hz}$ with a lower sweep rate of $0.25 \mathrm{~Hz} \mathrm{~s}^{-1}$ were used. The precise values of the observed frequencies were found to be slightly dependent on the sweep rate. In a series of separate experiments a factor of 2 change in sweep rate was found to shift the frequencies for a given volume by up to $\pm 5 \%$ for the higher modes. For comparison purposes we also observed oscillations on similar types of substrates using sessile droplets of (conducting) water without any powder coating (Figure 1b).

The profiles of the droplets were captured in silhouette illumination using an SVSI MemView high-speed camera and still images analyzed using the NIH Image J image processing software. By composing a stack of still images from a sequence and then creating a $z$-projection to provide a layered view, nodes could be identified around the surface of the droplets/marbles. Figure 1c illustrates the first resonance for a small $5 \mu \mathrm{L}$ volume liquid marble. In this image, the contact line appears to be mobile rather than pinned, although this is difficult to confirm visually because of the small contact area; the presence of the wire electrode does not appear to cause much distortion. Figure 1d shows a similar volume droplet in resonance on the flat hydrophobic surface. By analyzing amplitude changes in a region close to the electrode wire, resonant frequencies could be identified from the image stack. Performing a $z$-axis profile plot of this region, defined by a rectangular box selection typically located to the left- or right-hand side of the apex of the droplet gave mean gray scale values for the selected area throughout the sweep, which reached peak variance at points of peak amplitude of oscillation, thus defining resonant frequencies.

To determine the surface tension of the liquid marbles, we increased the volume of liquid in a marble and measured the maximal height. At small volumes the marble is spherical with a small flat spot (due to gravity) where it contacts the substrate, but as the volume increases, it changes to a puddle shape with a maximal height that tends asymptotically to twice the capillary length. ${ }^{14,17}$ Figure 2 shows the marble height as a function of the radius for the range of volumes used in the oscillation experiments; more extensive data for the same system are given in ref 17 . The transition from the spherical to puddle regime, illustrated by the inset pictures, is shown, and the limiting value of the puddle height provides an estimate of the surface tension within error limits of $52 \pm 2 \mathrm{mN} \mathrm{m}^{-1}$.

\section{Results and Discussion}

Resonance Spectra. Liquid marbles behave as nonwetting droplets and are completely mobile with no contact angle hysteresis. Although the contact angle of a liquid marble is $180^{\circ}$, for small marbles a flat spot with finite contact radius, $r$, at the interface with the substrate is induced due to gravity. This contact radius is given by $r=(2 / 3)^{1 / 2} \kappa R_{\mathrm{o}}^{2}$, where $R_{\mathrm{o}}$ is the radius of a 


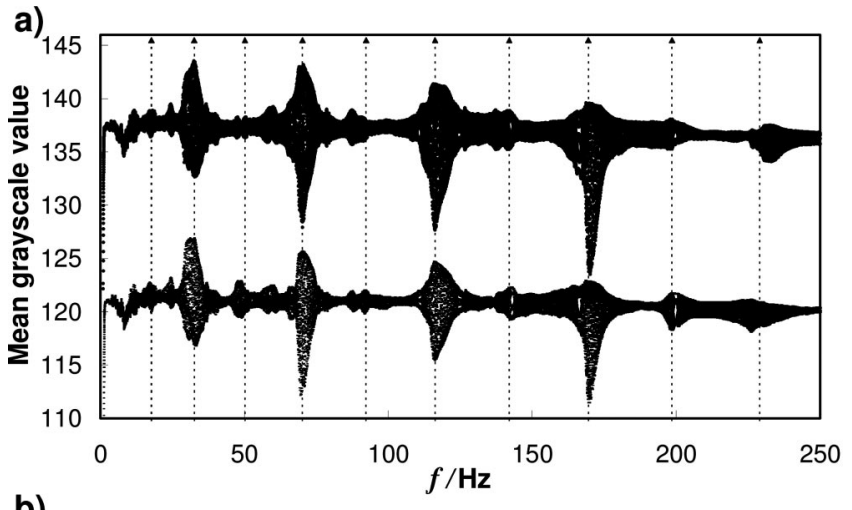

b)

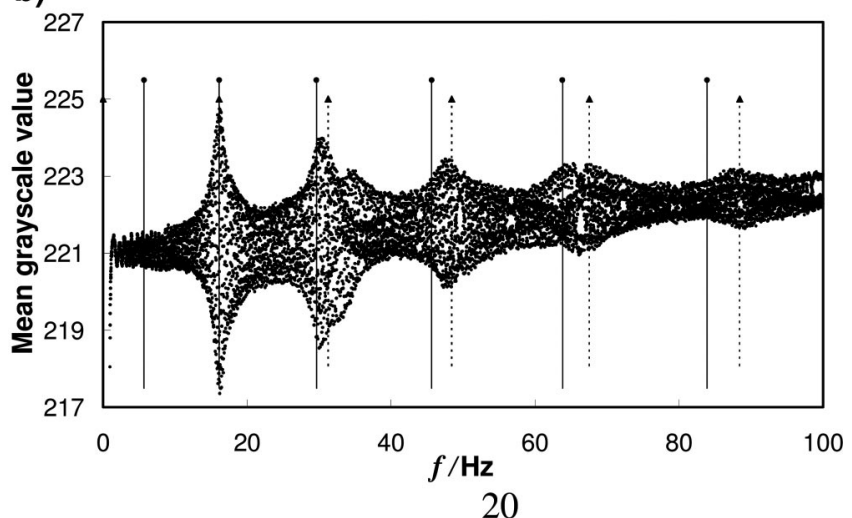

Figure 3. Example of the $z$-axis profile data used to locate resonant frequencies showing a mean gray scale value within a rectangular box selection at an antinode close to the electrode wire for (a) a $100 \mu \mathrm{L}$ liquid droplet (data from the right-hand side and left-hand side of the electrode wire are shown by the upper and lower curves) and (b) a liquid marble of the same volume. In (a), the vertical dotted lines are single-parameter fits to the capillary wave model. In (b), the vertical dotted lines are fits to the free fluid sphere droplet model using an $n(n-1)(n+2)$ mode dependence and the solid vertical lines are from the capillary wave model using an $n^{3}$ mode number dependence.

sphere containing the same volume of liquid and the capillary length is $\kappa^{-1}=\left(\gamma_{\mathrm{LV}} / \rho g\right)^{1 / 2} ; g=9.81 \mathrm{~ms}^{-2}$ is the acceleration due to gravity. ${ }^{13,14}$ When we applied the ac voltage with the frequency sweep to the liquid marbles, we observed two distinct types of motion. At low frequencies the mode was an up and down type motion. ${ }^{5,6}$ This was not localized to a single frequency, but occurred over a range of frequencies. As the frequency was increased, this motion was transformed into a resonant oscillation with a nodal pattern around the droplet periphery; the precise resonance frequency was dependent on the marble volume. As the volume increased, so did the number of modes observed in the wide-band sweep; up to 14 resonances were observed. The up and down type of motion was not observed for the sessile droplet on a planar surface, but resonant oscillations were observed.

Figure 3a shows an example of the $z$-axis profile data from the image stack of a $100 \mu \mathrm{L}$ sessile droplet during a wide-band sweep experiment. The upper curve corresponds to observations from the right-hand side of the contact wire used to apply the ac electrowetting voltage, and the lower curve is the data from the left-hand side of the contact wire; the $y$-axis gives the gray scale value in arbitrary units (an offset has been applied to separate the two data sets for display). Both sets of data show a sequence of sharp peaks with smaller and broader intermediate peaks; the change in amplitude of these peaks in Figure $3 \mathrm{a}$ is partially due to the position of the maxima relative to the fixed position of the rectangular box selection region. Figure $3 \mathrm{~b}$ shows equivalent

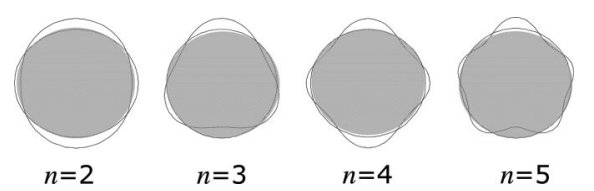

Figure 4. Schematic illustrations of shape modes for freely oscillating spherical droplets (side-view profile).

data for a comparable volume liquid marble. In this case, the major peaks with the sequence are not accompanied by any obvious intermediate modes.

Model for Immobile and Mobile Contact Line Modes. Immobile and mobile contact line type behaviors of vibrated sessile droplets have previously been identified by Noblin et al., who labeled them type I and type II modes, respectively. ${ }^{20}$ The transition between these types of behavior depends upon the magnitude of contact angle hysteresis and the amplitude of vibration. In their experiments using sessile droplets of water on a polystyrene substrate with excitation from below using a loudspeaker, they observed pure type I immobile contact line beahavior. In our case, small $5 \mu \mathrm{L}$ sessile droplets on the Teflon AF1600 hydrophobic planar surface appeared to always undergo resonances with a mobile contact line (Noblin type II), which is consistent with the observations reported by Oh et al., who also used Teflon AF1600. ${ }^{19}$ For larger $100 \mu \mathrm{L}$ droplets the situation was more complicated with both mobile and immobile contact line behavior observable at similar frequencies. For example, the first major peak in Figure 3a occurring at $33 \mathrm{~Hz}$ visually appeared to correspond to an immobile contact line, but at a few hertz lower frequency this was preceded by a mobile contact line. In the case of the liquid marble, which is perfectly nonwetting, contact angle hysteresis is completely absent and a mobile contact line (Noblin type II) behavior is expected. After the experiment, the marble remained mobile, with no liquid penetrating between the powder grains and pinning it to the surface, and could be removed from the surface by a light current of air, suggesting the liquid marble remained intact and possessed a mobile contact line.

Resonant frequencies of freely suspended and perfectly spherical vibrated droplets can be described by eq 1 . In the side profile view, the shape modes can be described by a combination of Legendre polynomials as shown schematically in Figure 4. A recent theoretical analysis of oscillations of a substratesupported hemispherical droplet has considered the cases of a fixed contact line and a constant contact angle. ${ }^{21}$ For a freely sliding contact line it was suggested that the natural frequencies should coincide with the natural frequencies of even oscillation modes for a spherical droplet. For axisymmetric vibrations of a spherical-cap-shaped droplet with a fixed contact line, eq 1 becomes

$$
f_{n}^{2}=\frac{\gamma_{L V}}{4 \pi^{2} \rho R^{3} \Lambda_{n}}
$$

where the eigenvalues $\Lambda_{n}$ are dependent on the contact angle. ${ }^{5}$ Smithwick and Boulet ${ }^{6}$ numerically tabulated values for the three lowest frequencies for contact angles from $100^{\circ}$ to $180^{\circ}$.

For oscillations of substrate-supported droplets, the resonant modes are due to stationary surface waves and the nodal patterns observed in the droplet images allow pseudowavelengths to be defined in terms of fractions of the droplet spherical circumference

(20) Noblin, X.; Buguin, A.; Brochard-Wyart, F. Eur. J. Phys. 2004, E14, 395-404.

(21) Lyubimov, D. V.; Lyubimov, T. P.; Shklyaev, S. V. Phys. Fluids 2006, 18,012101 . 
a)
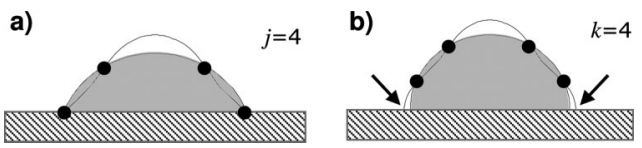

Figure 5. Pure oscillation modes for sessile droplets with (a) an immobile contact line (Noblin type I) and (b) a mobile contact line (Noblin type II). For type I modes the three-phase contact line corresponds to a node of the vibration, whereas for type II it is an antinode (indicated by the arrows).

(i.e., the profiles in Figure 1c,d). ${ }^{20}$ For 1-D capillary-gravity waves on a liquid bath of depth $h$ with wave vector $q_{n}=2 \pi / \lambda_{n}$, the angular frequency, $\omega_{n}$, is

$$
\omega_{n}^{2}=g q_{n}\left(1+\left(\frac{q_{n}}{\kappa}\right)^{2}\right) \tanh \left(q_{n} h\right)
$$

where $\kappa^{-1}=\left(\gamma_{\mathrm{LV}} / \rho g\right)^{1 / 2}$ is the capillary length. If the droplet is completely spherical and possesses the nodal shapes given by Figure 4 , the side-view perimeter length, $p=2 \pi R$, must accommodate a whole number, $n$, of wavelengths, $\lambda_{n}$. Assuming the bath is sufficiently deep, so that $\tanh \left(q_{n} h\right) \rightarrow 1$, and the capillary term is dominant, eq 3 approximates to

$$
f_{n}^{2} \approx \frac{n^{3} \gamma_{\mathrm{LV}}}{4 \rho \pi^{2} R^{3}}=\frac{n^{3} \gamma_{\mathrm{LV}}}{3 \pi \rho V}
$$

which is equivalent to eq 1 with $n(n-1)(n+2)$ replaced by $n^{3}$. Numerically, this gives a $\sim 10 \%$ underestimate for $n=2-7$, with this underestimate decreasing at higher values of mode number $n$.

In the case of a supported droplet, the Noblin type I mode can be understood by imposing nodes at the contact between the droplet and substrate to describe an immobile contact line with a constant contact radius (Figure 5a). For the Noblin type II mode, antinodes are imposed at the contact between the droplet and substrate to describe a completely mobile contact line with a constant contact angle (Figure 5b). For type I modes, the sideview profile perimeter, $p$, can accommodate a half-integer number of wavelengths, $p=(j-1) \lambda_{j} / 2$, where $j=2,3,4, \ldots$. The resonant frequencies are then described by

$$
\begin{aligned}
f_{j}^{2}=\frac{(j-1) g}{4 \pi p}\left(1+\left(\frac{(j-1) \pi}{p \kappa}\right)^{2}\right) \tanh \left(\frac{(j-1) \pi h}{p}\right) & \approx \\
& \frac{\pi(j-1)^{3} \gamma_{\mathrm{LV}}}{4 \rho p^{3}}(5)
\end{aligned}
$$

where the final approximation is the result for capillary-dominated waves on an infinitely deep bath. For type II modes, the sideview profile perimeter, $p$, can again accommodate a half-integer number of wavelengths, $p=k \lambda_{k} / 2$, where $k=2,3,4, \ldots$. In this case there is a quarter-wavelength distance between the last node and the antinode at the substrate on each side of the droplet. The resonant frequencies for modes with mobile contact lines are then described by

$$
f_{k}^{2}=\frac{k g}{4 \pi p}\left(1+\left(\frac{k \pi}{p \kappa}\right)^{2}\right) \tanh \left(\frac{k \pi h}{p}\right) \approx \frac{\pi k^{3} \gamma_{\mathrm{LV}}}{4 \rho p^{3}}
$$

where the final approximation is the result for capillary-dominated waves on an infinitely deep bath. In the Noblin type I and type II immobile and mobile contact line cases there are $j$ and $k$ nodes in each pattern, respectively. Equations 5 and 6 predict the same sequence of frequencies with the indexing related by $j=k+$ 1.
To understand the relationship between the nodal patterns for the Noblin type I and type II cases, consider the $j=4$ immobile contact line case from Figure 5a. This corresponds to three halfwavelengths around the perimeter, but three half-wavelengths can also be fitted into the $k=3$ mobile contact line case, which has three nodes separating the perimeter into two quarter wavelengths (one from the substrate to the first node above the substrate on each side) and one full wavelength between these two nodes with a node at the droplet apex splitting that wavelength itself into two half-wavelengths. In these resonant frequency sequences, odd number $j$ and $k$ always correspond to resonances possessing nodes at the apex of the droplet and so represent vibrations only possessing time-averaged axisymmetry. The analysis by Oh et al. indicates that the electrowetting excitation excites the even modes possessing an antinode at the droplet apex. ${ }^{19}$ To compare the free spherical fluid case to eq 6 , we rewrite eq 1 using $n(n-1)(n+2) \approx n^{3}$ and $p=2 \pi R$

$$
f_{n}^{2}=\frac{n(n-1)(n+2) \gamma_{\mathrm{LV}}}{4 \rho \pi^{2} R^{3}} \approx \frac{2 \pi n^{3} \gamma_{\mathrm{LV}}}{\rho p^{3}}
$$

This result appears to be a factor of 8 larger than the approximation in eq 6, but if we now restrict the values of $k$ to even values and write $k=2 n$ with $n=1,2,3,4, \ldots$, we recover the same result.

Fitting to Spectra for a Sessile Droplet. The dotted vertical lines in Figure 3a show a fit of eq 5 to the data for the $100 \mu \mathrm{L}$ sessile droplet using $j=3,4, \ldots, 12$, with the first major resonance occurring at $32.6 \mathrm{~Hz}$ corresponding to $j=4$; this fitting is equivalent to eq 6 using $k=2,3, \ldots, 11$. To determine the parameter $p$, the droplet profile has been assumed to be a spherical cap with contact angle $\theta$ so that the spherical radius is given by $R=$ $(3 V / \pi \beta(\theta))^{1 / 3}$, where $V$ is the droplet volume, $\beta(\theta)=2-3 \cos$ $\theta+\cos ^{3} \theta$ and the side-view perimeter is $p=2 R \theta$; this is only a rough approximation since at this volume the droplet has a flattened profile. The fit uses the standard values for the density and surface tension of water $\left(\rho=997 \mathrm{~kg} \mathrm{~m}^{-3}\right.$ and $\gamma_{\mathrm{LV}}=72.8$ $\mathrm{mN} \mathrm{m}{ }^{-1}$ ) together with the deposited volume $V$. The only adjustable parameter used in the fit is the effective contact angle, which has been set to $\theta=87^{\circ}$; this gives a perimeter length $10 \%$ lower than that estimated using a value for Teflon of $\theta \approx 115^{\circ}$. Factors influencing the fit, apart from the spherical model to convert volume to perimeter length, include the reduction in contact angle expected due to electrowetting, vibration inducing a receding angle, and possibly the effect of using the cubic dependence on the mode number rather than a more complex function of the mode number as occurs in the exact theory for a free spherical droplet.

Fitting to Spectra for a Liquid Marble. The solid vertical lines in Figure 3b show a fit of eq 7 for the mobile contact line to the data for the $100 \mu \mathrm{L}$ liquid marble; the first major peak at $16 \mathrm{~Hz}$ corresponds to $n=2$ with subsequent peaks at $n=3,4$, 5 , and 6 . The shape has been assumed to be a sphere so that $p$ $=2 \pi R$ and $R=(3 V / 4 \pi)^{1 / 3}$, and an $n^{3}$ mode dependence has been used. The fit uses the surface tension $\gamma_{\mathrm{LV}}=52 \mathrm{mN} \mathrm{m}^{-1}$ obtained from the maximal height of the liquid puddle (i.e., Figure 2) together with the deposited volume $V$. The only adjustable parameter used is the effective density, which has been taken as $\rho=1690 \mathrm{~kg} \mathrm{~m}^{-3}$. We emphasize that this is not a true density of the object, but is a method to set the numerical value of the overall combination $\gamma_{\mathrm{LV}} / \rho p^{3}$ using a single fitting parameter. This takes into account any error in estimating the marble's spherical radius by ignoring the flat spot of the marble at the contact with the substrate, any effects of the different densities of the powder coating and the encapsulated water, and any error in using the static value of $\gamma_{\mathrm{LV}}$. The dotted vertical lines show 


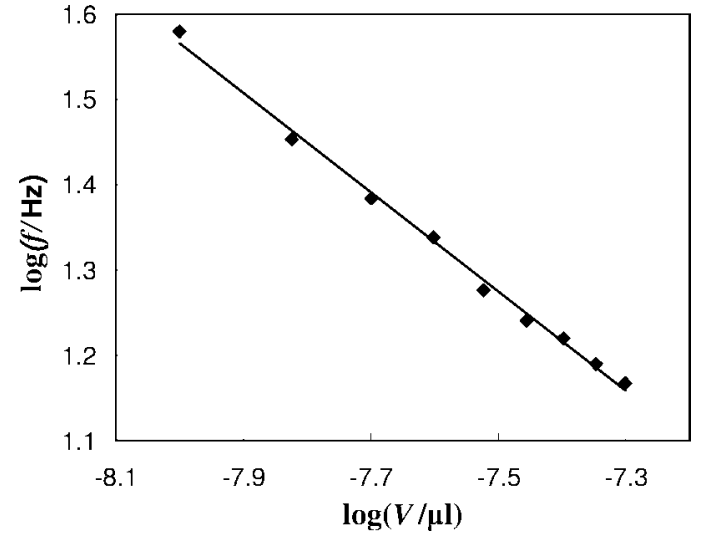

Figure 6. Change in the liquid marble resonant frequency for mode $n$ $=2$ as a function of the volume $(10-50 \mu \mathrm{L})$. The solid line is a fit and gives a -0.58 power law close to the theoretical value of -0.5 .

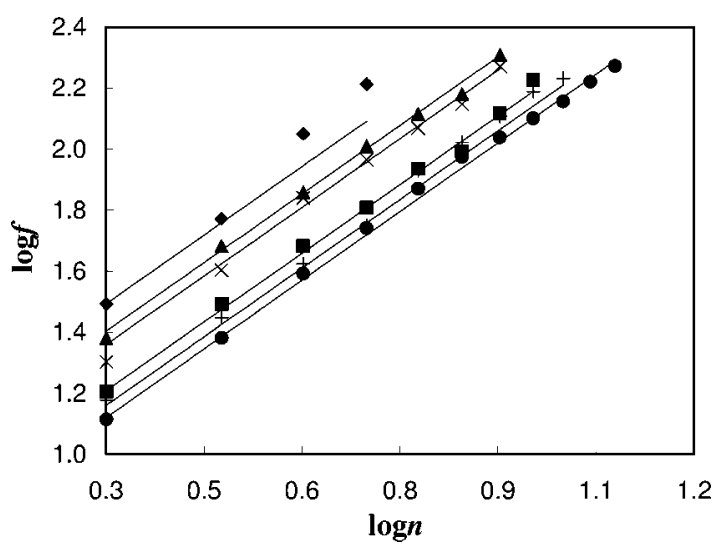

Figure 7. Frequency as a function of the mode number $(\log -\log$ representation) for liquid marbles of volumes $10 \mu \mathrm{L}(\bullet), 30 \mu \mathrm{L}(\mathbf{\Delta})$, $50 \mu \mathrm{L}(\times), 100 \mu \mathrm{L}(\mathbf{\square}), 125 \mu \mathrm{L}(+)$, and $150 \mu \mathrm{L}(\bullet)$. For the $50-150$ $\mu \mathrm{L}$ volumes the solid lines are fits to eq 7 using the same fitting parameter value of $\rho=1690 \mathrm{~kg} \mathrm{~m}^{-3}$ as for the data in Figure 3. For the 50 and $30 \mu \mathrm{L}$ data fitting values of $\rho=2300 \mathrm{~kg} \mathrm{~m}^{-3}$ and $\rho=4500 \mathrm{~kg} \mathrm{~m}^{-3}$ have been used.

the free fluid sphere model using the same parameters and the mode number combination $n(n-1)(n+2)$ rather than $n^{3}$; an improved fit could be obtained by slightly adjusting the value of $\rho$.

Figure 6 shows a test of the frequency dependence on the volume for the lowest mode $(n=2)$ for small liquid marbles with volumes from 10 to $50 \mu \mathrm{L}$. The data obey the power law $f \propto V^{-0.58}$, which is close to $f \propto V^{-1 / 2}$ expected from eq 6 . Figure 7 shows a test of the dependence of the resonant frequencies on the mode number for liquid marbles with volumes from 30 to $150 \mu \mathrm{L}$. The solid lines for $50,100,125$, and $150 \mu \mathrm{L}$ are all fits using eq 7 and $n=2,3,4, \ldots$ with exactly the same parameters as determined from the spectra for the $100 \mu \mathrm{L}$ liquid marble shown in Figure 3b. The fit for the smallest volume of $30 \mu \mathrm{L}$ required a larger effective density parameter of $\rho=2300 \mathrm{~kg} \mathrm{~m}^{-3}$ (i.e., a larger value of the combination $\gamma_{\mathrm{LV}} / \rho p^{3}$ ). It is unclear why this was the case, but it could be due to the limited accuracy arising in our system when dealing with the smallest volumes. For the $10 \mu \mathrm{L}$ volume liquid marble the small amplitude of oscillation caused difficulties in accurately identifying the modes, and this may account for the step in the trend of the data points for this volume; for completeness a solid line fit using $\rho=4560$ $\mathrm{kg} \mathrm{m}^{-3}$ has been fitted through the data.

As the volume of the liquid marble decreases, the effect from the electrode wire at the apex of the marble and the changes in volume due to evaporation will become relatively more important. The effects of shape distortion and the fundamental frequencies for large-volume marbles, whose shape tends to liquid puddles, could be further investigated using different camera perspectives, although this may require the use of transparent substrates. Resonances could also be excited using alternative methods, such as with a vertically or horizontally vibrating platform ${ }^{22-24}$ or by inducing vibrations using a loudspeaker. ${ }^{20}$ An alternative approach to using a liquid marble to investigate resonances of fluid spheres without using active levitation or microgravity might be to use a liquid droplet on a superhydrophobic surface. However, in this case very small feature sizes and high aspect ratios would be needed to prevent collapse into the Wenzel state. ${ }^{22-24}$

\section{Conclusion}

In this work we have used an electrowetting-on-dielectric approach to induce shape oscillations in liquid marbles. Resonant oscillations due to stationary capillary surface waves and which are accurately described by the theory for free spherical volumes of fluid have been observed. The liquid marble approach provides an idealized system for the study of the oscillations of a free liquid sphere without the need for any system to actively levitate the droplet. The system provides perfect nonwetting with zero contact angle hysteresis and a mobile contact line. These types of experiments could be extended to larger volume systems to study the capillary-to-gravity surface wave transition.

Acknowledgment. K.E. acknowledges the financial support of TÜBITAK. We also acknowledge the financial support of EPSRC and MOD/Dstl under Research Grant GR/S34168/01.

\section{LA803016F}

(22) Bormashenko, E.; Pogreb, R.; Whyman, G.; Bormashenko, Y.; Erlich, M. Appl. Phys. Lett. 2007, 90, 201917.

(23) Bormashenko, E.; Pogreb, R.; Whyman, G.; Erlich, M. Langmuir 2007, 23, 6501-6503.

(24) Bormashenko, E.; Pogreb, R.; Whyman, G.; Erlich, M. Langmuir 2007, $24,12217-12221$ 